\title{
Bulk Transport Properties and Exponent Inequalities for Random Resistor and Flow Networks
}

\author{
J. T. Chayes ${ }^{1}$ and L. Chayes ${ }^{2}$ \\ Laboratory of Atomic and Solid State Physics, Clark Hall, \\ Cornell University, Ithaca, NY 14853, USA
}

\begin{abstract}
The properties of random resistor and flow networks are studied as a function of the density, $p$, of bonds which permit transport. It is shown that percolation is sufficient for bulk transport, in the sense that the conductivity and flow capacity are bounded away from zero whenever $p$ exceeds an appropriately defined percolation threshold. Relations between the transport coefficients and quantities in ordinary percolation are also derived. Assuming critical scaling, these relations imply upper and lower bounds on the conductivity and flow exponents in terms of percolation exponents. The conductivity exponent upper bound so derived saturates in mean field theory.
\end{abstract}

\section{Introduction and Summary of Results}

The problem of bulk transport in random resistor networks has attracted a great deal of attention for almost two decades [1-22]. The simplest such network is one in which the bonds of a regular lattice are taken to be occupied independently by unit conductors with probability $p$, and vacant (i.e., of infinite resistance) with probability $1-p$. Interest in this model is a consequence of the suggestion, due to Ziman [1], Eggarter and Cohen [2,3] and others, that it provides a good description of the conductivity properties of (binary) inhomogeneous conductors when the disorder occurs on an intermediate scale. In particular, it was argued that the resistor network is capable of bulk conductivity if and only if $p$ exceeds the percolation threshold, $p_{c}$, of the lattice, i. e. that the resistive transition occurs at the percolation threshold.

Given the arguments for coincidence of these transition points, it was initially expected [1] that the bulk conductivity, $\sigma(p)$, should be proportional to the percolation probability, $P_{\infty}(p)$. However, this assumption was soon called into question by the table-top experiment of Last and Thouless [4]. If, following

\footnotetext{
${ }^{1}$ Research supported by the NSF under Grant No. DMR-8314625

${ }^{2}$ Research supported by the DOE under Grant No. DE-AC02-83ER13044
} 
Eggarter and Cohen [2], one introduces an effective mobility, $u(p)$, defined by:

$$
\sigma(p)=u(p) P_{\infty}(p),
$$

the Last and Thouless experiment suggested that $u(p)$ vanishes as $p \downarrow p_{c}$. Such behavior was also confirmed by Kirkpatrick $[5,6]$ in two- and three-dimensional simulations, and explicitly verified on the Bethe lattice by the exact solution of Stinchcombe [7] and the subsequent computation of the proper (bulk) conductivity by Straley [8].

Although the above results indicated that the conductivity vanishes with a larger critical exponent than that of the percolation probability, this alone did not rule out the possibility of a simple relationship between the exponents for conductivity and ordinary percolation quantities. However, later arguments suggested that the conductivity may depend on connectedness properties of percolation configurations which are not characterized by the standard critical exponents. Skal and Shklovskii [9], de Gennes [10] and others [11-13] used such arguments to propose a scaling relation for the conductivity in terms of the percolation correlation length, $\xi(p)$, and a new quantity, $L(p)$, which measures the relative length of strands connecting nodes in the "node-link" picture. If the critical exponents are "defined" by $\sigma(p) \sim\left|p-p_{c}\right|^{t}, P_{\infty}(p) \sim\left|p-p_{c}\right|^{\beta}, \xi(p) \sim\left|p-p_{c}\right|^{-v}$, and $L(p) \sim\left|p-p_{c}\right|^{-\zeta}$ as $p \downarrow p_{c}$, the Skal-Shklovskii-de Gennes scaling relation is

$$
t=(d-2) v+\zeta \text { for } d \leqq d_{c}=6 .
$$

Although there have been suggestions that the exponent $\zeta$ is either trivial (e.g., $\zeta=1$ for all $d$ [14]) or is related to standard percolation exponents (e.g., $\zeta=v$ in $d=2[15,16]$ or $\zeta=(d v-\beta) / 2$ for $d \leqq 6[17,18])$, the current evidence $[19,20]$ indicates that $\zeta$ is an "independent" exponent.

In spite of the theoretical interest, there has been remarkably little rigorous analysis of random resistor networks. Indeed, even the coincidence of the resistive transition and the percolation threshold is far from obvious. Not surprisingly, it is straightforward to establish that percolation is necessary for transport, in the sense that there is no conduction when $p$ is less than the percolation threshold (see the discussion in the beginning of Sect.3). However, in order to prove that $p_{c}$ is the resistive transition point, one must also show that when $p>p_{c}$, the percolation cluster provides sufficiently many connections to allow bulk transport. Thus a proof of the resistive transition involves a fairly detailed treatment of the connectedness properties of percolation configurations. The rigorous work to date is that of Grimmett and Kesten [23] (see also Kesten [24, Chap. 11]) who have shown that on the hypercubic bond lattice in two (or more) dimensions, bulk conductance occurs for $p>p_{c}^{[d=2]}=1 / 2$. To our knowledge, there has been very little rigorous consideration of the critical properties.

In this paper, we examine the random resistor problem and, a closely related issue, the random flow problem in all dimensions. In the latter case, one interprets the occupied bonds of the network as open channels capable of transmitting a fixed amount of fluid per unit time, and the vacant bonds as blocked channels. One then asks whether such a medium is capable of bulk transport of fluid, as characterized by a flow constant $c(p)$, which is the amount of fluid which drains through the medium per unit time per unit area. The distinction between the resistor and flow 
problems is best understood by noting that flow is not increased by the presence of loops of open channels intersecting open paths, in contrast to enhancement of conduction by the presence of additional conductors in parallel.

Our results on these models fall into two categories. First, we prove that percolation is sufficient for transport, in the sense that when $p$ exceeds an appropriately defined percolation threshold, there is bulk conductivity and bulk flow. Assuming certain technical conjectures on equivalence of transition points for the ordinary percolation problem, this extends the results of Grimmett and Kesten [23] to all dimensions.

Next, we consider the critical properties of bulk conductivity and flow, and relate these to the critical behavior of quantities in the ordinary percolation model. To explain our results, let us assume the existence of a "limiting" conductivity, $\sigma(p)$, and flow capacity, $c(p)$. (This is a technically very difficult and, at present, largely unresolved problem, which will be discussed in greater detail in the next section.) Under this assumption, our first result on the critical properties can be stated in the form

$$
\sigma(p) \leqq(d)\left[Q_{\infty}(p)\right] \leqq(p d)\left[P_{\infty}(p)\right]^{2}
$$

and

$$
c(p) \leqq Q_{\infty}(p) \leqq p\left[P_{\infty}(p)\right]^{2},
$$

where $Q_{\infty}(p)$ is the density of bonds with at least two disjoint connections to infinity, which can be interpreted as the "backbone" density of the infinite cluster. In particular, this implies that $\sigma(p) / P_{\infty}(p) \equiv u(p) \leqq(p d) P_{\infty}(p)$, so that the effective mobility must tend to zero at least as quickly as the percolation probability.

Assuming (the unproven conjecture) that the quantities under consideration exhibit critical scaling, and "defining" the exponents by $\sigma(p) \sim\left|p-p_{c}\right|^{t}, c(p)$ $\sim\left|p-p_{c}\right|^{f}, P_{\infty}(p) \sim\left|p-p_{c}\right|^{\beta}$, and $Q_{\infty}(p) \sim\left|p-p_{c}\right|^{q}$ as $p \downarrow p_{c}$, the results (1.3) and (1.4) imply the exponent inequalities

$$
t \geqq q \geqq 2 \beta
$$

and

$$
f \geqq q \geqq 2 \beta \text {. }
$$

It is worth noting that, while the inequality (1.5) is sharp enough to confirm predictions on the vanishing of the effective mobility, it is not a mean field bound. Indeed, on the Bethe lattice $q=2$ and $\beta=1$, while Straley [8] has shown that $t=3$.

Our second principal result on the critical properties was obtained by complementing (1.3) and (1.4) with lower bounds. We do this under the assumption that the infinite volume limiting quantities $\sigma(p)$ and $c(p)$ exist in an appropriate sense, and the further assumption that the flow constant $c(p)$ so obtained is, to a certain extent, independent to the sequence of hyperrectangles through which the infinite volume is taken. If we also assume critical scaling, we then obtain upper bounds on the transport exponents.

In order to explain our bound, we must first define the dual surface tension for ordinary bond percolation. To this end, consider the dual of the density $p$ bond percolation model, in which a dual $(d-1)$-cell is taken to be occupied whenever the bond traversal to it is vacant. By this correspondence, we obtain a $(d-1)$-cell Bernoulli percolation model at density $1-p$. Now consider the event that a loop 
composed of $(d-2)$-cells is spanned by an unbroken surface of these dual $(d-1)$ cells. If $p$ is large enough, it is easy to show that the probability of this event decays exponentially in the minimal $(d-1)$-cell area spanned by the loop (in $d \geqq 2$ ); the coefficient of exponential decay is, by definition, the surface tension $\tau(p)$. For example, in $d=3$ it has been shown [25] that whenever $p$ exceeds an appropriate percolation threshold, the probability that a loop of lattice edges is spanned by an unbroken surface of plaquettes decays exponentially in the minimal area of the loop, with coefficient of decay $\tau(p)>0$. Two dimensions is a somewhat degenerate case in which the above probability corresponds to the dual connectivity function, so that the "surface tension" is just half of the inverse of the dual correlation length.

It is generally assumed that the surface tension also exhibits critical scaling in the sense that $\tau(p) \sim\left|p-p_{c}\right|^{\mu}$ as $p \downarrow p_{c}$. In terms of the exponent $\mu$, our second bound on the conductivity may be written in the form

$$
t \leqq 2 \mu-q \leqq 2(\mu-\beta) \text {. }
$$

Now recall that the mean field value for $v$ is $1 / 2$. Using this and the "knowledge" that $d_{c}=6$, the mean field value $\mu=5 / 2$ is predicted by the Widom scaling relation $\mu=(d-1) v\left(d \leqq d_{c}\right) ; \mu=\left(d_{c}-1\right) v\left(d \geqq d_{c}\right)$. Recalling the mean field values $\beta=1$ and $t=3$, we see that (1.7) is actually a mean field bound. Indeed, the estimates used in the derivation of (1.7) provide some insight into the mechanism for bulk conductivity, and confirm the intuition underlying the scaling relation (1.2) in high dimension.

There are two ingredients in the derivation of (1.7) which may be of independent interest. The first is an inequality relating the resistance and flow problems:

$$
\sigma(p) \geqq(1 / d)\left[c^{2}(p) / Q_{\infty}(p)\right],
$$

or, in exponent language:

$$
t \leqq 2 f-q
$$

We expect that (1.9) saturates only in mean field.

The second ingredient in (1.7) is a bound relating the flow constant $c(p)$ and the dual surface tension $\tau(p)$. If these quantities have critical scaling, the bound implies that

$$
f \leqq \mu
$$

It is possible that this exponent inequality is sharp in all dimensions; indeed, if there is critical scaling, this can be proven for $d=2$ [26].

Finally, let us examine the bound $q \geqq 2 \beta$, which provides an additional inequality in both our upper and lower bounds on $t$. It turns out that saturation of this bound (i.e., $q=2 \beta$ ) is essentially a characterization of mean field behavior. Thus, we expect that in low dimension, the result $q \leqq t \leqq 2 \mu-q$ should be a lot stronger than the corresponding result in terms of $\beta$.

The outline of the paper is as follows. In Sect. 2, we define the problems under consideration. We also discuss the technical difficulties to which we have alluded concerning existence of the constants $\sigma(p)$ and $c(p)$. Our proof of bulk transport above an appropriate percolation threshold is given in Sect. 3, and critical properties are derived in Sect. 4. 


\section{Definitions and Statement of the Problem}

In this section, we define the random resistor and flow problems (precisely), and review some basic properties of ordinary percolation.

We begin by defining a resistor or flow network. This is done by specifying (1) a lattice, and (2) a distribution $\varrho$ of nonnegative random numbers assigned identically and independently to the elements of the lattice. For simplicity, we will only consider networks defined on the hypercubic bond lattice $\mathbb{B}_{d}$, which is by definition the set of all bonds between nearest neighbor pairs of sites on $\mathbb{Z}^{d}$. The random variable $\omega_{b} \in[0, \infty]$ assigned to $b \in \mathbb{B}_{d}$ represents the transport characteristics of the bond $b$; for the resistor network, each bond $b$ should be regarded as a separate resistor of resistance $\omega_{b}$; for the flow network, $\omega_{b}$ represents the maximum amount of fluid which can flow through the bond $b$ in a unit time. Thus our choice of the distribution $\varrho$ is dictated by the physics we wish to model. As discussed in the introduction, we are concerned with systems in which each bond has positive probability of not permitting transport, so we will later restrict to resistor networks with $\varrho(\infty)>0$, and flow networks with $\varrho(0)>0$. We denote by $\Omega$ the sample space (set of all realizations), and by $\mu_{e}$ the product measure on this space.

Bulk transport properties are determined by "measurements" (which turn out to be stationary random variables) that represent the response of the system to a stress applied at its boundary. It is convenient to consider large samples characterized by a single dimension $L$. Thus we denote by $\Lambda_{L}$ the cube:

$$
\Lambda_{L}=\left\{x \in \mathbb{Z}^{d} \mid 0 \leqq x_{1}, \ldots, x_{d} \leqq L\right\},
$$

and by $H_{L}(\omega)$ the restriction of the configuration $\omega \equiv\left(\omega_{b} \mid b \in \mathbb{B}_{d}\right) \in \Omega$ to those bonds $b \in \Lambda_{L}$ with both endpoints in $\Lambda_{L}$.

The resistance of the cube $\Lambda_{L}$ is determined by placing conducting plates on two opposing faces and measuring (or computing via Kirchoff's laws) the potential difference between the plates that is required to draw one unit of current.

The flow capacitance of $\Lambda_{L}$ can be measured by pumping fluid in through one face of the cube and determining the amount of fluid which flows through the opposing face per unit time. In order to compute the flow capacitance, we must consider all flow patterns which are consistent with $H_{L}(\omega)$. The flow patterns are constructed by first orienting the bonds $b \in \Lambda_{L}$, and then assigning to each of them a flux $f_{b}$ such that the collection of flux numbers satisfies current conservation at each vertex strictly inside the cube. Thus for each $x \in \Lambda_{L}$ with $0<x_{1}<L$, we require that

$$
\varphi_{x}=\sum_{b: x \in \partial b} v_{b} f_{b}
$$

must vanish. In the above expression, $v_{b}=+1$ if $b$ is oriented towards $x$, and $v_{b}=-1$ if $b$ is oriented away from $x$. Any such collection $\mathbf{f}=\left(f_{b} \mid b \in \Lambda_{L}\right)$ is called a flow pattern. A flow pattern $\mathbf{f}$ is said to be consistent with $H_{L}(\omega)$ if $\left|f_{b}\right| \leqq \omega_{b}$ for each $b \in \Lambda_{L}$. The flux $\Phi(\mathbf{f})$ of the flow pattern $\mathbf{f}$ is given by

$$
\Phi(\mathbf{f})=\sum_{x: x_{1}=L} \varphi_{x},
$$

which is the rate at which fluid accumulates at the right boundary. The flow capacitance of $\Lambda_{L}$ in configuration $\omega$ is computed by optimizing $\Phi$ over all flow patterns consistent with $H_{L}(\omega)$. 
It should be clear that the two measurements described above provide us with information on bulk transport properties: the first is the electrical resistance of the cube, and the second is the rate at which fluid flows through the cube. We will denote these random variables by $R_{L}(\omega)$ and $C_{L}(\omega)$, respectively. It is worth noting that these transport properties are distinct - both mathematically and physically from local transport characteristics. (For the resistor network, examples of the latter include the resistance between well-separated sites and the minimum resistance along any single path connecting a pair of sites.)

In the deterministic case (i.e., if $\varrho$ is a degenerate distribution concentrated on some $0<a<\infty), R_{L}$ and $C_{L}$ exhibit a characteristic (engineering) scaling:

$$
\begin{gathered}
R_{L} \sim a L^{-(d-2)}, \\
C_{L} \sim a L^{d-1} .
\end{gathered}
$$

For finite volumes, these formulas are not exact due to edge effects. This motivates the definition of rescaled variables:

$$
\begin{gathered}
r_{L}(\omega)=L^{d-2} R_{L}(\omega), \\
c_{L}(\omega)=L^{-(d-1)} C_{L}(\omega),
\end{gathered}
$$

which, in the deterministic case, converge to the transport coefficients (i.e., the resistivity and the flow capacity) as $L \uparrow \infty$.

One would also like to define meaningful transport coefficients in the random case. This requires some type of convergence of $r_{L}(\omega)$ and $c_{L}(\omega)$ as $L \uparrow \infty$. For various reasons, one would like to show a.s. convergence to a constant. Unfortunately, this seems to be a very difficult problem. In the case of resistor networks, it follows from the results of Kipnis and Varadhan [27] that if $\varrho$ is supported on a bounded interval (so that, in particular, there are no infinite resistors), then $r_{L}$ converges in $L^{2}(d \mu)$. (Similar results were first established for a continuum resistance problem by Papanicolaou and Varadhan [28] and Golden and Papanicolaou [29].) The only progress on existence of transport coefficients for the types of distributions we will consider here has been made by Kesten $[30,31]$ who showed that, for the flow network, if $\varrho(0)$ is sufficiently small, then $c_{L}$ converges a.s. to a constant.

In this paper, we will not concern ourselves with existence of a limit, focusing instead on nontriviality of a limit, should it exist. This question is meaningful only if, with positive probability, any given bond is incapable of transport [i. e., $\varrho(\infty)>0$ in the resistance network, and $\varrho(0)>0$ in the flow network]. The essential features of this system are best exhibited in the case in which all bonds capable of exhibiting transport have $\omega_{b}=1$, the so-called Bernoulli problem. Many of the results we will derive can be extended with some obvious modifications to more general cases.

For the Bernoulli system, we will denote by $p$ the probability that $\omega_{b}=1$. We will have occasion to refer to the bonds with $\omega_{b}=1$ as occupied bonds. In the opposite case $-\omega_{b}=0$ for the flow network, and $\omega_{b}=\infty$ for the resistor network we will often refer to the bonds as vacant. The idea is then to study the system as a function of $p$. For this, let us recall some basic results of percolation theory.

The percolation transition in the Bernoulli system on $\mathbb{B}_{d}$ concerns the formation of an infinite connected component of occupied bonds. It is relatively 
straightforward to establish the existence (or lack), with probability one, of an infinite cluster at high (respectively, low) values of $p$. Denoting by $P_{\infty}(p)$ the probability that the origin is connected to an infinite cluster of occupied bonds, the above considerations motivate the definition of the percolation threshold:

$$
p_{c}=\inf \left\{p \mid P_{\infty}(p)>0\right\} .
$$

We shall occasionally use the notation $p_{c}(d)$ for the above quantity when we want to explicitly demonstrate its dimensional dependence.

There are several other possible definitions of a percolation threshold which are believed to coincide with (2.8). First, let $\chi(p)$ denote the expected size of the occupied connected cluster of the origin. Then we can define the transition point

$$
\pi_{c}=\inf \{p \mid \chi(p)=\infty\} .
$$

Although it is expected that $p_{c}=\pi_{c}$ for all $d$, this has only been proven for $d=2$ [32] (and, of course, is known trivially for $d=1$ ).

Another threshold may be defined by considering half-space percolation. Let $\bar{P}_{\infty}(p)$ denote the probability that the origin is connected to infinity by a path of occupied bonds which lies entirely in the half-space $\left\{x_{1} \geqq 0\right\}$. The half-space threshold is given by

$$
\bar{p}_{c}=\inf \left\{p \mid \bar{P}_{\infty}(p>0\} .\right.
$$

Again, one expects that $\bar{p}_{c}=p_{c}$ for all $d$, but this has only been proven for $d=2$ $[33,34]$.

A fourth threshold is suggested by the analysis of [25]. Consider the restriction of the bonds of $\mathbb{B}_{d}$ to the "slab"

$$
S_{k}=\left(\mathbb{Z}^{+}\right)^{2} \times\{0, \ldots, k\}^{d-2},
$$

where $\left(\mathbb{Z}^{+}\right)^{2}$ denotes the positive quadrant of $\mathbb{Z}^{2}$. Let us denote by $\hat{p}_{c}^{[k]}$ the percolation threshold for this bond lattice (i. e., $\hat{p}_{c}^{[k]}$ is the smallest value of $p$ above which there is positive probability that the origin of $S_{k}$ is connected to an infinite cluster of occupied bonds within $\left.S_{k}\right)$. It is natural to conjecture [25] that $p_{c}(d)$ coincides with

$$
\hat{p}_{c}^{\infty}=\lim _{k \rightarrow \infty} \hat{p}_{c}^{[k]} .
$$

Such results are known to hold in Ising systems [39].

\section{The Bulk Transport Transition}

The purpose of this section is to relate the resistive and flow critical points to the percolation threshold. Our principal result (Theorem 3.6) is that whenever $p>\hat{p}_{c}^{\infty}$, there is bulk transport in the sense that, as $L \uparrow \infty, r_{L}$ is bounded away from infinity and $c_{L}$ is bounded away from zero w. p. 1 . Modulo the conjecture $p_{c}=\hat{p}_{c}^{\infty}$, this establishes that percolation is sufficient for bulk conduction and flow. As mentioned in the introduction, it is already known that percolation is necessary for bulk transport, so that (assuming $p_{c}=\hat{p}_{c}^{\infty}$ ) this identifies the percolation threshold as the bulk transition point. 


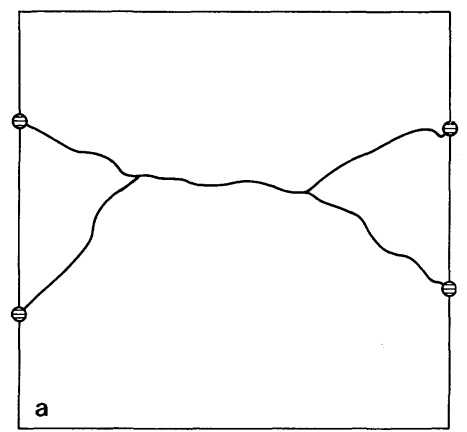

Fig. 1. A configuration with one disjoint path

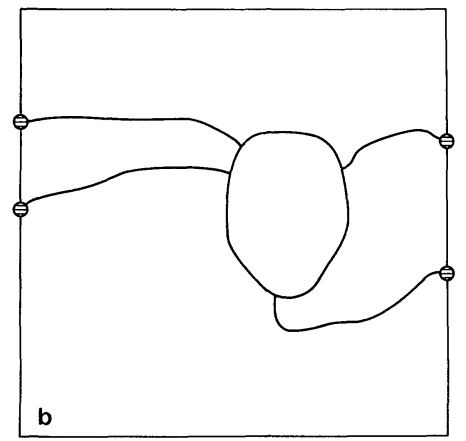

A configuration with two disjoint paths

For completeness, let us first review the results on necessity. It is easy to show that $\operatorname{Prob}\left(r_{L}=\infty\right)$ and $\operatorname{Prob}\left(c_{L}=0\right)$ converge exponentially to one as $L \uparrow \infty$ whenever $p<\pi_{c}$. The nontrivial ingredient is to establish that these quantities converge (not necessarily exponentially) to one even if $p<p_{c}$. For this, it suffices to use the a. s. bound $\liminf _{L \rightarrow \infty} r_{L} \geqq p / P_{\infty}(p)$, derived by Kesten [24, Theorem 11.3]. The reader is referred to Proposition 4.5 for a strengthened form of this bound.

Let us now turn to the question of sufficiency. Here, we must first determine conditions under which a given configuration will have bulk transport, and then show that such conditions are satisfied with large probability.

It is obvious that the transport properties of a (finite) cube are related to how well the opposing faces of the cube are connected. In this regard, it is not the number of sites on one face connected to the opposing face which determines the transport characteristics, but rather the number of such connections which occur through disjoint (i.e., independent) paths (see Fig. 1). Indeed, in the Bernoulli flow problem, it is seen that this number is exactly the flow capacitancy, $C_{L}(\omega)$, of the cube. Thus for a given configuration to exhibit bulk flow, there must be $O\left(L^{d-1}\right)$ disjoint connections between opposing faces. For the resistance problem, the statement can be made as an inequality.

To prove the above assertion, the first ingredient we require is a "physically obvious" inequality which allows us to bound from above the resistance of a given circuit by neglecting the contribution of any individual resistor. Unfortunately, as with many "correlation inequalities," the proof of this fact (which was apparently known in the last century) is less obvious than one initially expects. An elegant formulation of the statement was proved by Bott and Duffin [35]:

Proposition 3.1 [35]. Let $v_{1}, \ldots, v_{m}$ denote a set of vertices, with each pair $\left(v_{i}, v_{j}\right)$ joined by a resistor of resistance $\omega_{i j} \equiv 1 / \sigma_{i j}$. Let $a$ and $b$ be any two points in the circuit, and let $R_{a b}=R_{a b}\left(\left\{\sigma_{i j}\right\}\right)$ denote the total resistance between these points. Then $\forall(k, l)$

$$
d R_{a b} / d \sigma_{k l}=-f^{2}\left(\left\{\sigma_{i j}\right\}\right)
$$

where $f$ is an algebraic function of the $\left\{\sigma_{i j}\right\}$, the numerator and denominator of which are linear in each of the $\sigma_{i j}$. 
Remark. By obvious continuity, this extends to the lattice cases we consider here simply by taking the relevant $\sigma_{i j}$ 's to zero. Integration of the expression (3.1) then shows that removal of any finite resistor from the circuit can only raise the resistance between any pair of points.

Given this inequality, it is not hard to relate the resistance to the flow problem, as in the proposition below. Although a proposition of this form is implicit in certain theorems of Kesten (see, e. g., Eq. (11.81) in [24]), we provide an explicit bound which we later improve for our analysis of the critical properties.

Proposition 3.2. $\forall \omega \in \Omega$,

$$
r_{L}(\omega) \leqq \kappa_{L}(d) / c_{L}^{2}(\omega),
$$

where $\kappa_{L}(d)(\sim d)$ is the number of bonds per unit volume in a cube of side $L$.

Proof. If $C_{L}(\omega)=0$, we are done. Otherwise, there are $C_{L}(\omega)>0$ mutually disjoint paths of occupied bonds connecting the left face of the cube to the right. Although this collection is not unique, we may enumerate some such collection: $s_{1}, \ldots, s_{C_{L}}$. Let us further denote by $\left|s_{j}\right|$ the number of bonds in the $j^{\text {th }}$ path. Observe that $\left|s_{j}\right|$ is also the resistance between the two ends of this path.

By Proposition 3.1, the total resistance $R_{L}(\omega)$ of the cube is bounded above by the resistance of these paths alone. The latter quantity is easily computed, since the paths are in parallel:

$$
R_{L}^{-1} \geqq \sum_{j=1}^{C_{L}}\left|S_{j}\right|^{-1}=C_{L} \sum_{j=1}^{C_{L}}\left(C_{L}\left|S_{j}\right|\right)^{-1} .
$$

The bound can be degraded even further using the Jensen inequality:

so that

$$
R_{L} \leqq C_{L}^{-2} \sum_{j=1}^{C_{L}}\left|s_{j}\right|
$$

$$
r_{L} \leqq\left[\kappa_{L}(d) / c_{L}^{2}\right]\left(\left[\kappa_{L}(d) L^{d}\right]^{-1} \sum_{j=1}^{C_{L}}\left|s_{j}\right|\right) .
$$

The term in brackets above is just the fraction of bonds in the cube which participate in the formation of the paths $\left\{s_{j} \mid j=1, \ldots, C_{L}\right\}$, and hence is bounded above by one.

It should now be clear that both transport problems may be analyzed by estimating the number of disjoint crossings of a cube of side $L$. This is the subject of the remainder of this section. Our principal estimate is the following.

Lemma 3.3. Suppose $p>\hat{p}_{c}^{\infty}$. Then there exist constants $\lambda_{1}, \lambda_{2}>0$ such that

$\operatorname{Prob}_{p}\left(\exists\right.$ at least $\lambda_{1} L^{d-1}$ disjoint left-to-right crossings of $\left.\Lambda_{L}\right) \geqq 1-e^{-\lambda_{2} L^{d-1}}$ for L sufficiently large.

Remark. It should be noted that the probabilistic estimate of Lemma 3.3 exhibits optimal scaling; indeed with probability exceeding

$$
(1-p)^{(L+1)^{(d-1)}}
$$


there are no crossings of $\Lambda_{L}$. In this regard, it is worth commenting that our approach differs from that employed elsewhere in that we first establish the correct form of the scaling for the probability that there is nonzero transport (i. e., there is at least one left-to-right crossing), and then use this to show that (with similarly scaling probability), there is, in fact, bulk transport. Thus, as a corollary to Lemma 3.3, we have that for $p>\hat{p}_{c}^{\infty}$, the cube crossing probability tends to one exponentially in $L^{d-1}$. On the other hand, it is well known that for $p<\pi_{c}$, this probability tends to zero exponentially in $L$. This implies that for $d \geqq 3$, the cube crossing probability has quite different scaling behaviors above and below threshold.

In order to prove Lemma 3.3, we will need two items, both of which were derived in [25].

Proposition 3.4 [25, Lemma 4.9]. Consider the "square"

$$
t_{L}^{[k]}=\left\{x \in\left(\mathbb{Z}^{+}\right)^{2} \times\{0, \ldots, k\}^{d-2} \mid 0 \leqq x_{1}, x_{2} \leqq L\right\},
$$

and denote by $T_{L}^{[k]}$ the probability that there is a ("hard way") crossing of $t_{L}^{[k]}$ from the left boundary $\left(x_{1}=0\right)$ to the right boundary $\left(x_{1}=L\right)$ by a path of occupied bonds. If $p>\hat{p}_{c}^{[k]}$, then there exists a positive constant $m(p, k)$ such that

$$
T_{L}^{[k]} \geqq 1-e^{-m(p, k) L}
$$

for L sufficiently large.

Remark. The derivation of Proposition 3.4 in [25] was only for $d=3$, but it extends in an obvious fashion to $d \geqq 4$.

The second item we will use concerns the stability of certain events which occur with large probability.

Definition. Let $A \subset \Omega$ be an event. The $N$-stability sphere of $A$, denoted by $\mathrm{I}^{N}(A) \subset A$, is the set of configurations in which the event $A$ occurs even if the configuration is altered at as many as $N$ bonds. In other words, if

$$
s^{N}(\omega)=\left\{\omega^{\prime} \in \Omega\left|\sum_{b}\right| \omega_{b}-\omega_{b}^{\prime} \mid \leqq N\right\}
$$

is the set of configurations which differ from $\omega$ on no more than $N$ bonds, then

$$
\mathrm{I}^{N}(A)=\left\{\omega \in A \mid s^{N}(\omega) \subset A\right\} .
$$

It turns out that the most useful applications of the stability sphere occur in the context of positive events:

Definition. Consider the partial order on $\Omega$ defined by the condition that $\omega>\omega^{\prime}$ if $\forall b \in \mathbb{B}_{d}, \omega_{b} \geqq \omega_{b}^{\prime}$. An event is said to be positive or increasing in the sense of HarrisFKG $[36,37]$ if its indicator function is nondecreasing with respect to this partial order.

The content of the following proposition is that, if a positive event occurs with large probability, then at a slightly higher bond density, its stability sphere is also large. 
Proposition 3.5 [25, Lemma 4.2]. Let $A$ be a positive event. Then

$$
\left.\operatorname{Prob}_{p} \mathbf{I}^{N}(A)\right) \geqq 1-(p / \varepsilon)^{N}\left[1-\operatorname{Prob}_{p-\varepsilon} \mathrm{I}^{N}(A)\right] .
$$

We now derive our key estimate:

Proof of Lemma 3.3. Let $\varepsilon \equiv\left(p-\hat{p}_{c}^{\infty}\right) / 2$ and take $p^{\prime} \equiv(p-\varepsilon)>\hat{p}_{c}^{\infty}$. Then there is a $k$ large enough so that $p^{\prime}>\hat{p}_{c}^{[k]}$. Now take $L \gg k$ and divide (most of) the cube $\Lambda_{L}$ into $[L / k+1]^{d-2}$ disjoint translates of the rectangle $t_{L}^{[k]}$, where $[L / k+1]$ is the largest integer smaller than $(L / k+1)$. By independence of events in the different translates, and by Proposition 3.4, we obtain, at density $p^{\prime}$

$$
\operatorname{Prob}_{p^{\prime}}\left(\exists \text { a left-to-right crossing of } \Lambda_{L}\right) \geqq 1-e^{-m\left(p^{\prime}, k\right) L[L / k+1]^{d-2}} .
$$

However, $\mathrm{I}^{N}\left(\exists\right.$ a left-to-right crossing of $\left.\Lambda_{L}\right)$ is just the event that there are more than $N$ disjoint crossings of $\Lambda_{L}$. Thus, by Proposition 3.5,

$\operatorname{Prob}_{p}\left(\exists>N\right.$ disjoint left-to-right crossings of $\left.\Lambda_{L}\right) \geqq 1-(p / \varepsilon)^{N} e^{-m(p-\varepsilon, k) L[L / k+1]^{d-2}}$

$$
\equiv 1-(p / \varepsilon)^{N} e^{-g(p-\varepsilon, k) L^{d-1}},
$$

with $g(p-\varepsilon, k)>0$. Taking $N=\lambda_{1} L^{d-1}$ with $\lambda_{1}$ chosen small enough so that $|\log (p / \varepsilon)| \lambda_{1}<g(p-\varepsilon, k)$, Lemma 3.3 is established.

The conclusions of this section are summarized below.

Theorem 3.6. Suppose $p>\hat{p}_{c}^{\infty}$. Then there exists constants $\lambda_{1}, \lambda_{2}>0$ such that, for $L$ sufficiently large,

$$
c_{L} \geqq \lambda_{1} \quad \text { and } \quad r_{L} \leqq \kappa_{L}(d) / \lambda_{1}^{2}
$$

with probability exceeding $1-e^{-\lambda_{2} L^{d-1}}$. In particular,

and

$$
\liminf _{L \rightarrow \infty} c_{L} \geqq \lambda_{1}
$$

w.p.1.

$$
\limsup _{L \rightarrow \infty} r_{L} \leqq d / \lambda_{1}^{2}
$$

Proof. The result for the flow network follows from Lemma 3.3 and the observation that $C_{L}$ is precisely the number of disjoint crossings of $\Lambda_{L}$. The resistance result then follows from Proposition 3.2.

\section{Critical Exponent Inequalities for Resistance and Flow}

The results of the previous section establish that, modulo the equivalence of critical points, the presence of an infinite cluster is sufficient to ensure nontrivial transport coefficients. As mentioned previously, it was already known [24] that the coefficients are trivial in the absence of an infinite cluster. However, as emphasized in the introduction, and as should be apparent from the proofs of the previous section, a strong degree of connectedness is required for bulk transport.

The purpose of this section is to quantify the above notion by examining different degrees of connectedness, thereby deriving bounds on the transport coefficients in terms of various percolation probabilities. Assuming scaling limits of all these quantities (which, as a prerequisite, assumes the existence of well- 
defined transport coefficients) and the equivalence of critical points, these bounds imply inequalities among the critical exponents.

Just as one can distinguish the questions of necessity and sufficiency of percolation for bulk transport, there are distinct questions of connectedness which one must address to obtain upper and lower bounds on the critical exponents. Lower bounds on the exponents are analogous to the question of necessity, and are again the easier case. In order to derive lower bounds, one must show that there are certain bonds which do not contribute to transport. In order to obtain upper bounds on the exponents, we examine the number of chains that contribute to transport. We already know from the sufficiency proof of the last section that this number must be $O\left(L^{d-1}\right)$; here, we obtain bounds on the exponents by controlling the coefficient of $L^{d-1}$.

\subsection{Lower Bounds on the Transport Exponents}

As explained above, lower bounds on the transport exponents are obtained by showing that not all occupied bonds contribute to transport. Of course, we may immediately rule out finite clusters of occupied bonds which (eventually) cannot serve to connect opposing faces of a cube. However, as first noted by Last and Thouless [4], even if a bond is connected to infinity, it may not contribute to overall transport. Indeed, by current conservation, it is seen that any bond which is connected to infinity, but which can be disconnected by the removal of a single other bond, cannot carry current across a sufficiently large box. Bonds which have this property are often called "dangling bonds;" the rest of the infinite cluster is called the "backbone." Let us define a backbone density.

Consider the event, which we will denote by $B_{\infty}(b)$, that $(1)$ the bond $b$ is occupied, and (2) the two endpoints of $b$ are connected to infinity by disjoint paths. If $B_{\infty}(b)$ occurs, we will say that $b$ is in the backbone cluster. The backbone density is just the probability of this event for some fixed bond $b$, i.e., $Q_{\infty}(p)$ $\equiv \operatorname{Prob}_{p}\left(B_{\infty}(b)\right)$.

It is worth noting that $B_{\infty}(b)$ may also be expressed as the intersection

$$
B_{\infty}(b)=\bigcap_{N=1}^{\infty} B_{N}(b)
$$

where $B_{N}(b)$ is the event that $b$ is occupied and that its endpoints are connected to disjoint clusters of size exceeding $N$. Furthermore, if $Q_{N}(p)=\operatorname{Prob}_{p}\left(B_{N}(b)\right)$, then by continuity (of the measure)

$$
Q_{\infty}(p)=\lim _{N \rightarrow \infty} Q_{N}(p)
$$

The relevance of the backbone density to transport is the content of the following theorem.

\section{Theorem 4.1.}

(a) $\underset{L \rightarrow \infty}{\limsup } c_{L} \leqq Q_{\infty}(p) \quad$ w.p.1.

(b) $\liminf _{L \rightarrow \infty} r_{L} \geqq(1 / d)\left[Q_{\infty}(p)\right]^{-1} \quad$ w.p.1. 
Remark. Although the proofs of (a) and (b) are conceptually similar, that of (b) requires certain additional arguments. Thus we treat (a) and (b) separately.

Proof of (a). Consider the cube $\Lambda_{L}$, and let $\mathbb{P}_{L}$ denote the bonds of $\Lambda_{L}$ traversal to the hyperplane $x_{1}=[L / 2]+1 / 2$. (Note that this hyperplane is basically the midplane of the cube.) Let $\omega \in \Omega$ and $N<L / 2$. It is clear that the number of disjoint left-to-right crossings of $\Lambda_{L}$ in $\omega$ cannot exceed the number of bonds of $\mathbb{P}_{L}$ which are occupied and connected to two disjoint clusters of size greater than $N$, i. e.

$$
C_{L}(\omega) \leqq \sum_{b \in \mathbb{P}_{L}} \mathbb{1}_{B_{N}(b)}(\omega)
$$

Thus $\forall \omega \in \Omega$ and $N<L / 2$

$$
c_{L}(\omega) \leqq((L+1) / L)^{d-1}(L+1)^{-(d-1)} \sum_{b \in \mathbb{P}_{L}} \mathbb{1}_{B_{N}(b)}(\omega) .
$$

By the ergodic theorem, the right-hand side converges a. s. to $Q_{N}(p)$. Evidently $\forall N$

$$
\limsup _{L \rightarrow \infty} c_{L} \leqq Q_{N}(p) \quad \text { w.p.1. }
$$

Claim (a) is established by taking $N \uparrow \infty$.

In order to prove (b), we first determine the minimum resistance of a circuit with a fixed number of resistors.

Proposition 4.2. Let $n$ be a positive integer and consider all configurations of $n$ unit resistors in the cube $\Lambda_{L}$ (i.e., all configurations with $\left|H_{L}(\omega)\right|=n$ ). The minimum resistance of the cube for any such configuration has the lower bound

$$
R_{L}^{[n]} \equiv \min _{\omega:\left|H_{L}(\omega)\right|=n} R_{L}(\omega) \geqq L^{2} / n .
$$

Remark. Whenever $n=j L$ for some integer $j$, this bound has the interpretation that the minimum resistance is obtained by organizing the resistors into as many disjoint chains as possible. Indeed, in these cases, the bound is optimal.

Proof. The resistance in any configuration $H_{L}(\omega)$ is only decreased by replacing all resistors in the transverse hyperplanes $x_{1}=1,2, \ldots, L-1$ by superconductors. This has the effect of collapsing the circuit into a linear circuit of $L+1$ points.

Let $k_{j}(\omega), j=1, \ldots, L$, be the number of resistors in $H_{L}(\omega)$ which connect the hyperplanes $x_{1}=j-1$ and $x_{1}=j$. Then, by the above reasoning,

$$
R_{L}(\omega) \geqq \sum_{j=1}^{L} k_{j}^{-1}(\omega)
$$

Using the Jensen inequality, we obtain

$$
R_{L}(\omega) \geqq L^{2} / \sum_{j=1}^{L} k_{j}(\omega) .
$$

But $\sum k_{j}(\omega)$ cannot exceed the total number of resistors in the circuit, which we now take to be $n$. This establishes the desired result.

Proof of Theorem 4.1 (b). Let $\omega \in \Omega$ and denote by $H_{L} *(\omega) \subset H_{L}(\omega)$ the configuration consisting only of those bonds in $H_{L}(\omega)$ which are connected to the left and 
right faces of $\Lambda_{L}$ by disjoint paths of occupied bonds. By current conservation, the resistance in configuration $H_{L} *(\omega)$ is identical to that in $H_{L}(\omega)$. Thus if $n_{L}(\omega)$ $=\left|H_{L} *(\omega)\right|$ is the number of bonds in $H_{L} *(\omega)$, Proposition 4.2 implies

$$
R_{L}(\omega) \geqq L^{2} / n_{L}(\omega) .
$$

Let us obtain an upper bound on $n_{L}(\omega)$. To this end, take $N<L / 2$. Observe that any bond that contributes to $n_{L}(\omega)$, and that has $x_{1}$-coordinate between $N$ and $L-N$, must be connected to two disjoint chains exceeding $N$. Thus

$$
n_{L}(\omega) \leqq \kappa_{L}(d) N L^{d-1}+\sum_{b \in \Lambda_{L}} \mathbb{1}_{B_{N}(b)}(\omega)
$$

where, as defined in Proposition 3.2, $\kappa_{L}(d)$ is the number of bonds per unit volume in a cube of side $L$.

By (4.8) and (4.9), we have

Evidently, $\forall N$

$$
r_{L}(\omega) \geqq\left[\kappa_{L}(d)\right]^{-1} /\left\{N / L+\left[\kappa_{L}(d) L^{d}\right]^{-1} \sum_{b \in \Lambda_{L}} \mathbb{1}_{B_{N}(b)}(\omega)\right\}
$$

$$
\liminf _{L \rightarrow \infty} r_{L} \geqq(1 / d)\left[Q_{N}(p)\right]^{-1} \quad \text { w.p.1 . }
$$

The theorem is established by taking $N \uparrow \infty$.

In order to draw conclusions concerning the critical exponents, we must make several assumptions. Explicitly, we must assume that the transport coefficients exist, that both these coefficients and the backbone density vanish as $p \downarrow p_{c}$ (hence the assumption of coincidence of critical points), and that the vanishing of these quantities can be described by power laws, i. e. as $p \downarrow p_{c}$

$$
\begin{gathered}
c(p) \sim\left|p-p_{c}\right|^{f}, \\
r(p) \sim\left|p-p_{c}\right|^{-t}, \\
Q_{\infty}(p) \sim\left|p-p_{c}\right|^{q},
\end{gathered}
$$

where $\sim$ is meant in the sense of (say) logs and limits. Under the above assumptions, Theorem 4.1 implies the following:

Corollary 4.1. The critical exponents $f, t$, and $q$, should they exist, satisfy the inequalities $f \geqq q$ and $t \geqq q$.

\subsection{Bounds on the Backbone Density Exponent}

Since our bounds on the transport exponents are in terms of the backbone density, it is of some interest to relate the latter to other quantities in percolation theory. In this subsection, we obtain upper and lower bounds on $Q_{\infty}(p)$ in terms of the percolation probability, $P_{\infty}(p)$, and the half-space percolation probability, $\bar{P}_{\infty}(p)$. Our derivation of the upper bound (which is the one we will subsequently use) requires the van den Berg-Kesten inequality [38] for events that occur disjointly.

Definition. Let $A$ be an event, and take $S \subset \mathbb{B}_{d}$. The event $A$ is said to occur on the set $S$, denoted by $A_{\mid S}$, if $A$ occurs and is not destroyed by altering bonds in the complement of $S$. (In other words, $\omega \in A_{\mid S}$ if, by viewing the configuration 
restricted to $S$, we can determine that $A$ has occurred.) Two events $A$ and $B$ are said to occur disjointly, denoted by $A \circ B$, if there are disjoint sets on which $A$ and $B$ occur:

$$
A \circ B=\left\{\omega \in A \cap B \mid \exists S, T \subset \mathbb{B}_{d}, S \cap T=\emptyset, \omega \in A_{\mid S} \cap B_{\mid T}\right\} .
$$

The van den Berg-Kesten inequality provides an upper bound on $\operatorname{Prob}(A \circ B)$ : Proposition 4.3 (van den Berg-Kesten inequality [38]). If $A$ and $B$ are positive events, then

$$
\operatorname{Prob}(A \circ B) \leqq \operatorname{Prob}(A) \operatorname{Prob}(B) \text {. }
$$

We now derive our bounds on the backbone density:

Theorem 4.4. $p\left[\bar{P}_{\infty}(p)\right]^{2} \leqq Q_{\infty}(p) \leqq p\left[P_{\infty}(p)\right]^{2}$.

Proof. The lower bound is almost a triviality. It follows from the observation that $B_{\infty}(b)$ must occur for some bond $b$ along (say) the $x_{1}$-axis if (1) the bond $b$ is occupied; (2) the left endpoint of $b$ is connected to infinity in the half-space that lies to the left of $b$; and (3) the right endpoint of $b$ is connected to infinity in the halfspace to the right of $b$. Since these three events are independent, we have

$$
Q_{\infty}(p) \geqq p\left[\bar{P}_{\infty}(p)\right]^{2} .
$$

The upper bound is also easy. It follows from the observation that $B_{\infty}(b)$ occurs if (1) $b$ is occupied; (2) there is a path, which makes no use of $b$, from the left endpoint of $b$ to infinity; and (3) there is a path from the right endpoint of $b$ to infinity which makes use of neither $b$ nor any bonds along the path described in (2). Hence by the van den Berg-Kesten inequality

$$
Q_{\infty}(p) \leqq p\left[P_{\infty}(p)\right]^{2} .
$$

Assuming that both $P_{\infty}(p)$ and $\bar{P}_{\infty}(p)$ exhibit critical scaling as $p \downarrow p_{c}$ (and also that $\bar{p}_{c}=p_{c}$ ):

we have

$$
\begin{aligned}
& P_{\infty}(p) \sim\left|p-p_{c}\right|^{\beta}, \\
& \bar{P}_{\infty}(p) \sim\left|p-p_{c}\right|^{\beta^{*}},
\end{aligned}
$$

Corollary 4.4. The critical exponents $q, \beta$, and $\beta^{*}$, should they exist, satisfy the inequality

$$
2 \beta^{*} \geqq q \geqq 2 \beta \text {. }
$$

Combining Theorem 4.1 and the upper bound of Theorem 4.4, we obtain:

\section{Proposition 4.5.}

(a) $\limsup _{L \rightarrow \infty} c_{L} \leqq p P_{\infty}^{2}(p) \quad$ w.p.1.

(b) $\liminf _{L \rightarrow \infty} r_{L} \geqq(1 / d)\left[p P_{\infty}^{2}(p)\right]^{-1} \quad$ w.p.1.

In particular, if the quantities $c(p), r(p)$, and $P_{\infty}(p)$ exhibit critical scaling as $p \downarrow p$, then the exponents $f, t$, and $\beta$, should they exist, satisfy

and

$$
f \geqq 2 \beta
$$

$$
t \geqq 2 \beta \text {. }
$$


Remarks. (1) Proposition 4.5(b) represents an improvement of Kesten's bound [24, Theorem 11.3], and thus also implies that $\liminf _{L \rightarrow \infty} r_{L}=\infty$ w.p.1 whenever $p<p_{c}$.

(2) Although the bounds (4.21) and (4.22) have the advantage of relating the transport exponents to a conventional percolation exponent, it is likely that these are weaker than the bounds of Corollary 4.1; indeed, we expect that $q>2 \beta$ unless the model is in mean field.

\subsection{Upper Bounds on the Transport Exponents}

We now derive upper bounds on the transport exponents. This is done in two steps. First, we improve the estimate of Proposition 3.2, thereby deriving a new inequality relating the resistance and flow networks. Under assumptions analogous to those of previous sections, this inequality provides us with an upper bound on the resistivity exponent, $t$, in terms of the flow capacity exponent, $f$. Thus in addition to relating the networks, this inequality reduces the question of upper bounds on $t$ and $f$ to that of an upper bound on $f$. Under certain additional assumptions, we derive such a bound in our final theorem.

For technical reasons, our theorem relating the resistance and flow networks is more conveniently expressed in terms of the conductivity, $\sigma_{L} \equiv 1 / r_{L}$, than in terms of the resistivity.

Theorem 4.6. $\underset{L \rightarrow \infty}{\liminf } \sigma_{L} \geqq(1 / d)\left[Q_{\infty}(p)\right]^{-1} \limsup _{L \rightarrow \infty} c_{L}^{2} \quad$ w.p.1.

Proof. This theorem is just an improvement of the estimate of Proposition 3.2. In particular, the bound (3.5) may be written in the form

$$
\sigma_{L} \geqq\left\{L^{-d} \sum_{j=1}^{C_{L}}\left|s_{j}\right|\right\}^{-1} c_{L}^{2}
$$

Thus it suffices to show that, in the limit $L \rightarrow \infty$, the coefficient of $c_{L}^{2}$ is bounded below by $(1 / d)\left[Q_{\infty}(p)\right]^{-1}$. This, however, follows immediately from the proof of Theorem 4.1(b) and the observation that $\forall \omega \in \Omega$

$$
\sum_{j=1}^{C_{L}}\left|s_{j}\right| \leqq n_{L}(\omega)
$$

where, as defined previously, $n_{L}(\omega)$ is the total number of bonds in $\omega$ connected to the left and right faces of $\Lambda_{L}$ by disjoint paths.

If the limiting coefficients $\sigma(p)$ and $c(p)$ were to exist, and if these coefficients, as well as $P_{\infty}(p)$ and $Q_{\infty}(p)$, exhibited critical scaling as $p \downarrow p_{c}$, Theorem 4.6 and Corollary 4.5 would imply

Corollary 4.6. The critical exponents $t, f, q$, and $\beta$, should they exist, satisfy the inequality

$$
t \leqq 2 f-q \leqq 2 f-2 \beta \text {. }
$$

Next, we relate the flow capacity to the dual surface tension. As discussed in the introduction, the dual $(d-1)$-cell percolation model at density $1-p$ is equivalent to the original bond percolation model at density $p$. Now let $\gamma$ denote a loop (i. e., a surface without boundary) composed of $(d-2)$-cells of the direct lattice. We define 
$W \gamma$ to be the event that $\gamma$ is spanned, in the $\mathbb{Z}_{2}$ sense, by a surface of $(d-1)$-cells. That is, $W \gamma$ is the event that, among all occupied dual $(d-1)$-cells, there is a subset, the $\mathbb{Z}_{2}$ boundary of which is the loop $\gamma$. We denote by $\langle W \gamma\rangle_{p}$ the probability of the event $W \gamma$, and by Area $(\gamma)$ the minimum area [i.e., $(d-1)$-cell volume] of a surface spanning the loop $\gamma$.

If $\left\{\gamma_{i}\right\}$ is a sequence of (say, rectangular) loops, it was shown in [25] that

$$
\lim _{i \rightarrow \infty}\left[-\left(\log \left\langle W \gamma_{i}\right\rangle_{p}\right) / \operatorname{Area}\left(\gamma_{i}\right)\right] \equiv \tau(p)
$$

exists. We call $\tau(p)$ the dual surface tension. As a corollary to the proof of (4.25), one also obtains the a priori bound

$$
\left\langle W \gamma_{i}\right\rangle_{p} \leqq \exp \left[-\tau(p) \operatorname{Area}\left(\gamma_{i}\right)\right] \quad \forall i
$$

Our final result (Theorem 4.7) relates the flow capacity $c(p)$ to the dual surface tension $\tau(p)$. In contrast to our previous results, which could be stated in a meaningful form in the absence of a proof of existence of the transport coefficients, Theorem 4.7 requires a rather strong assumption on the flow capacity. To explain this assumption, take $\delta>0$ and consider a hyperrectangular of size $L \times \ldots \times L \times \delta L$. Let us denote by $C_{L}^{(1, \ldots, \delta)}(\omega)$ the amount of fluid per unit time which flows through the rectangle in the $x_{d}$ ("easy") direction in configuration $\omega$. One expects that $\forall \delta>0$,

$$
\lim _{L \rightarrow \infty} C_{L}^{(1, \ldots, \delta)}(\omega) / L^{d-1}=c(p) \quad \text { w.p. } 1 \text {. }
$$

Although this is stronger than a.s. existence of the flow capacity for hypercubes [since here we require $c(p)$ to be independent of $\delta$ ], it is not an unreasonable assumption. Indeed, Kesten [31] has established even stronger results (in $d=3$ ) for $p$ in a Peierls regime; in particular, (4.27) holds for rectangles of the form $\left(L, L, L_{3}\right)$ provided that $L^{-2+r} \log L_{3} \rightarrow 0$ as $L \rightarrow \infty$ for some $r>0$.

Theorem 4.7. If the flow capacity exists in the sense of (4.27), then

$$
c(p) \geqq \sup _{\varepsilon}[\tau(p-\varepsilon) /|\log (p / \varepsilon)|] .
$$

Proof. For the purposes of clarity, we will first give the proof for $d=3$, and then indicate the necessary modifications for other dimensions.

Take $\delta>0$. Consider a rectangle of dimensions $L \times L \times \delta L$, which we will denote by $\Lambda_{L}^{(1,1, \delta)}$. Let $\gamma_{L}$ denote the $L \times L$ loop which is the boundary of the bottom face of $\Lambda_{L}^{(1,1, \delta)}$.

We claim that if there is no bottom-to-top flow through $\Lambda_{L}^{(1,1, \delta)}$ (i. e., if the event $C_{L}^{(1,1, \delta)}=0$ occurs), and if the four vertical walls of $\Lambda_{L}^{(1,1, \delta)}$ are plated with occupied plaquettes, then there is a surface spanning the loop $\gamma_{L}$. By independence of these two events, this means

$$
\left\langle W \gamma_{L}\right\rangle_{p} \geqq(1-p)^{4 \delta L^{2}} \operatorname{Prob}_{p}\left(C_{L}^{(1,1, \delta)}=0\right) .
$$

To prove the above claim, we will show that a surface spanning $\gamma_{L}$ can be produced from the event $C_{L}^{(1,1, \delta)}=0$ and the plating of the vertical walls. Consider then configurations in which these two events occur. Let $R$ denote the connected component of all occupied bonds in $\Lambda_{L}^{(1,1, \delta)}$ which emanate from the bottom face of 
$\Lambda_{L}^{(1, \overline{1}, \delta)}$. (By connected component in $\Lambda_{L}^{(1,1, \delta)}$, it is understood that the connections must occur entirely within $\Lambda_{L}^{(1,1, \delta)}$.) Let $\partial R$ denote the (occupied) dual plaquettes in the boundary of $R$. In general, $\partial R$ will contain several isolated components. However, in the configurations we are considering, there are no paths of occupied bonds inside $\Lambda_{L}^{(1,1, \delta)}$ emanating from the bottom face of $\Lambda_{L}^{(1,1, \delta)}$ which pierce any of the other faces. In particular, this means that there is no circuit capable of supporting nonzero flux through the bottom face which interlocks $\gamma_{L}$. Thus a subset of $\partial R$ must be a surface with $\mathbb{Z}_{2}$ boundary $\gamma_{L}$. This establishes the claim (4.28).

Next, we note that the a priori bound (4.26) implies

$$
\left\langle W \gamma_{L}\right\rangle_{p} \leqq \exp \left[-\tau(p) L^{2}\right] .
$$

Combining this with (4.28), and defining $e^{-\alpha(p)} \equiv(1-p)^{4}$, we have

$$
\operatorname{Prob}_{p}\left(C_{L}^{(1,1, \delta)}>0\right)=1-\operatorname{Prob}_{p}\left(C_{L}^{(1,1, \delta)}=0\right) \geqq 1-e^{-[\tau(p)-\alpha(p) \delta] L^{2}} .
$$

Now, just as in the proof of Lemma 3.3, we note that the $N$-stability sphere of the event $C_{L}^{(1,1, \delta)}>0$ is the event $C_{L}^{(1,1, \delta)}>N$. Thus, by Proposition 3.5, we have

$$
\operatorname{Prob}_{p}\left(C_{L}^{(1,1, \delta)}>N\right) \geqq 1-(p / \varepsilon)^{N} e^{-[\tau(p-\varepsilon)-\alpha(p-\varepsilon) \delta] L^{2}} .
$$

Again, we take $N=\lambda L^{2}$. Now, however, we choose

$$
\lambda(p)=\sup _{\varepsilon}[\tau(p-\varepsilon) /|\log (p / \varepsilon)|]
$$

Since $\delta>0$ is arbitrary, the assumption (4.27) implies

the desired result.

$$
c(p) \geqq \lambda(p),
$$

The modifications for $d \neq 3$ are straightforward. In general, we consider the hyperrectangle $\Lambda_{L}^{(1,1, \ldots, 1, \delta)}$, the bottom face of which is an $L \times L \times \ldots \times L(d-1)$ dimensional hypercube composed of $(d-1)$-cells. The boundary of this face, which we again denote by $\gamma_{L}$, is a closed $L \times L \times \ldots \times L$ "loop" composed of $(d-2)$-cells. In this case, (4.28) becomes

$$
\left\langle W \gamma_{L}\right\rangle_{p} \geqq(1-p)^{2(d-1) \delta L^{d-1}} \operatorname{Prob}_{p}\left(C_{L}^{(1,1, \ldots, 1, \delta)}=0\right),
$$

which again follows from the observation that, in configurations in which all of the $(d-1)$-cells along the $2(d-1)$ "walls" are occupied and in which the event $C_{L}^{(1,1, \ldots, 1, \delta)}=0$ occurs, the boundary of the connected component of occupied bonds in $\Lambda_{L}^{(1,1, \ldots, 1, \delta)}$ emanating from the bottom face of $\Lambda_{L}^{(1,1, \ldots, 1, \delta)}$ consists of (isolated pieces and) a surface, composed of $(d-2)$-cells, with $\mathbb{Z}_{2}$ boundary precisely $\gamma_{L}$. The a priori bound now gives

$$
\left\langle W \gamma_{L}\right\rangle_{p} \leqq \exp \left[-\tau(p) L^{d-1}\right] .
$$

Thus, provided that we define $e^{-\alpha(p)} \equiv(1-p)^{2(d-1)}$, we need only change $L^{2}$ to $L^{d-1}$ in the rest of the proof.

If we also assume that $c(p)$ has critical scaling given by (4.12), and that as $p \downarrow p_{c}$

$$
\tau(p) \sim\left|p-p_{c}\right|^{\mu}
$$


and in (say) the sense of logs and limits, then Theorem 4.7 implies

Corollary 4.7. The critical exponents $f$ and $\mu$, should they exist, satisfy the inequality

$$
f \leqq \mu .
$$

It is possible that $f=\mu$ for all $d \geqq 2$. In fact, such an assertion can be proven [26] in $d=2$, where $\tau(p)$ is simply half of the inverse dual correlation length.

Combining Corollary 4.7 with Corollary 4.6, we obtain an upper bound on the resistivity exponent:

Corollary 4.7*. The critical exponents $t, \mu, q$, and $\beta$, should they exist, satisfy the inequality

$$
t \leqq 2 \mu-q \leqq 2(\mu-\beta) \text {. }
$$

As discussed in the introduction, if we use the mean field value for $\mu$ given by Widom scaling, we see that this bound saturates in mean field.

Acknowledgements. It is a pleasure to thank M. Aizenman, B. Hughes, H. Kesten, and C. M. Newman for useful discussions. We would also like to thank Harvard University, where we were NSF postdoctoral fellows while part of this work was done. Finally, we appreciate the hospitality and support of J. Lewis and the Dublin Institute for Advanced Study, where some crucial inequalities were derived.

\section{References}

1. Ziman, J.M.: J. Phys. C1, 1532 (1968)

2. Eggarter, T.P., Cohen, M.H.: Phys. Rev. Lett. 25, 807 (1970)

3. Eggarter, T.P., Cohen, M.H.: Phys. Rev. Lett. 27, 129 (1971)

4. Last, B.J., Thouless, D.J.: Phys. Rev. Lett. 27, 1719 (1971)

5. Kirkpatrick, S.: Phys. Rev. Lett. 27, 1722 (1971)

6. Kirkpatrick, S.: Rev. Mod. Phys. 45, 574 (1973)

7. Stinchcombe, R.B.: J. Phys. C6, L1 (1973)

8. Straley, J.P.: J. Phys. C10, 3009 (1977)

9. Skal, A.S., Shlovskii, B.I.: Fiz. Tekh. Poluprov. 8, 1586 (1974) [Sov. Phys. - Semicond. 8, 1029 (1975)]

10. de Gennes, P.G.: J. Physique Lett. 37, L1 (1976)

11. Harris, A.B., Fisch, R.: Phys. Rev. Lett. 77, 796 (1977)

12. Dasgupta, C., Harris, A.B., Lubensky, T.C.: Phys. Rev. B17, 1375 (1978)

13. Stephen, M.: Phys. Rev. B 17, 4444 (1978)

14. Wallace, D.J., Young, A.P.: Phys. Rev. B17, 2384 (1978)

15. Levinstein, M.E., Shur, M.S., Efros, A.L.: Zh. Eksp. Teor. Fiz. 69, 2203 (1975) [Sov. Phys. JETP 42, 1120 (1975)]

16. Straley, J.P.: J. Phys. C13, 819 (1980)

17. Alexander, S., Orbach, P.: J. Physique Lett. 43, L625 (1982)

18. Harris, A.B., Lubensky, T.C.: J. Phys. A16, L365 (1983)

19. Harris, A.B., Kim, S., Lubensky, T.C.: Phys. Rev. Lett. 53, 743 (1984) [see also Rammal, R., Lemieux, M.-A., Trembly, A.-M.S.: Phys. Rev. Lett. 54, 1087 (1985)]

20. Lobb, C.J., Frank, D.J.: Phys. Rev. B30, 4090 (1984)

21. Straley, J.P.: J. Phys. C15, 2333 (1982)

22. Hughes, B., Prager, S.: In: Mathematics and physics of disordered media. Lecture Notes in Mathematics, Vol. 1035. Berlin, Heidelberg, New York: Springer 1983

23. Grimmett, G., Kesten, H.: Z. Wahrscheinlichkeitstheor. Verw. Geb. 66, 335 (1984) 
24. Kesten, H.: Percolation theory for mathematicians. Boston: Birkhäuser 1982

25. Aizenman, M., Chayes, J.T., Chayes, L., Fröhlich, J., Russo, L.: Commun. Math. Phys. 92,19 (1983)

26. Chayes, J.T., Chayes, L., Durrett, R.: On the critical behavior of the two-dimensional first passage problem (preprint)

27. Kipnis, C., Varadhan, S.R.S.: Central limit theorem for additive functional of reversible Markov processes and applications to simple exclusions. Commun. Math. Phys. (in press)

28. Papanicolaou, G.C., Varadhan, S.R.S.: In: Colloquia Math. Soc. János Bolyai 27. Random Fields, Estergom (Hungary), p. 835. Amsterdam: North-Holland 1979

29. Golden, K., Papanicolaou, G.: Commun. Math. Phys. 90, 473 (1983)

30. Kesten, H.: First passage percolation and a higher dimensional generalization (preprint) (1985)

31. Kesten, H.: Surfaces with minimal random weights and maximal flows: A higher dimensional generalization of first passage percolation (preprint) (1985)

32. Kesten, H.: Commun. Math. Phys. 74, 41 (1980)

33. Russo, L.: Wahrscheinlichkeitstheor. Verw. Geb. 43, 39 (1978)

34. Seymour, P.D., Welsh, D.J.A.: Ann. Discrete Math. 3, 227 (1978)

35. Bott, R., Duffin, R.J.: Trans. Am. Math. Soc. 74, 99 (1953)

36. Harris, T.E.: Proc. Camb. Phil. Soc. 56, 13 (1960)

37. Fortuin, C., Kastelyn, P., Ginibre, J.: Commun. Math. Phys. 22, 89 (1981)

38. van den Berg, J., Kesten, H.: Inequalities with applications to percolation and reliability (to appear in Adv. Appl. Prob.)

39. Aizenman, M.: Phys. Rev. Lett. 54, 839 (1985)

Communicated by A. Jaffe

Received December 19, 1985 